\title{
Effects of educational intervention on nurses' knowledge, attitudes, and behavioral intentions toward supplying artificial nutrition and hydration to terminal cancer patients
}

\author{
Li-Shan Ke • Tai-Yuan Chiu $\cdot$ Wen-Yu Hu • Su-Shun Lo
}

Received: 2 November 2007 / Accepted: 13 February 2008 /Published online: 12 March 2008

(C) Springer-Verlag 2008

\begin{abstract}
Introduction This study aimed to investigate the effects of educational intervention on nurses' knowledge, attitudes, and behavioral intentions regarding supplying artificial nutrition and hydration (ANH) to terminal cancer patients. Materials and methods A quasi-experimental design was adopted. A structured questionnaire evaluated the effects of educational intervention. From April to June 2005, 88 nurses
\end{abstract}

\section{L.-S. Ke}

Department of Nursing, Taipei Veterans General Hospital,

Taipei, Taiwan

T.-Y. Chiu

College of Medicine, National Taiwan University,

Taipei, Taiwan

T.-Y. Chiu

Department of Family Medicine,

National Taiwan University Hospital,

Taipei, Taiwan

W.-Y. Hu $(\bowtie)$

School of Nursing, College of Medicine,

National Taiwan University,

1 Ren-Ai Rd Sect. 1, Jhongjheng District,

Taipei 100, Taiwan

e-mail: weyuhu@ntu.edu.tw

W.-Y. Hu

Department of Nursing, National Taiwan University Hospital,

Taipei, Taiwan

S.-S. Lo

College of Medicine, National Yang-Ming University,

Taipei, Taiwan

\section{S.-S. Lo}

Department of Surgery, Taipei Veterans General Hospital,

Taipei, Taiwan were enrolled in the gastroenterology, general surgery, and intensive care unit of Taipei Veterans General Hospital in Taiwan. The nurses were randomly assigned into experimental and control groups in equal numbers (44 nurses in each group). After the experimental and control groups completed the pretest, the experimental group participated in a 50-min lecture. Both groups received a post-test 2 weeks after the lecture.

Results This study showed that prior to educational intervention, nurses have possessed experiences of ANH use in routine caring for terminal cancer patients. However, due to the lack of knowledge about supplying ANH to terminal cancer patients, the nurses trended toward the negative behavioral intention, although they realized the burdens of $\mathrm{ANH}$ in these patients. After educational intervention, mean scores of knowledge, attitudes and behavioral intentions of the experimental group increased significantly $(z=-5.255$, $p<0.001 ; t=-5.191, p<0.001 ; z=-3.274, p \leqq 0.001)$. Mean score changes of knowledge and attitude between these two groups reached significant differences $(t=-7.306, p<0.001$; $t=-4.165, p<0.001$ ), but no significant difference was observed in the mean score change of behavioral intention $(z=-1.943, p>0.05)$.

Conclusion The educational intervention remarkably improved nurses' knowledge and attitudes regarding supplying terminal cancer patients with ANH. As for the changes in the behavioral intentions, it requires long-term moral and ethical training and communication. The results of this research emphasized the importance of educational interventions, which should be considered seriously in future reference nursing education program.

Keywords Artificial nutrition and hydration .

Terminal cancer patients $\cdot$ Knowledge $\cdot$ Attitudes .

Behavioral intentions 


\section{Introduction}

Cancer leads the top ten causes of death in Taiwan. In 2006, 37,998 individuals died from cancer, an incidence of 166.5 per 100,000 population, reflecting $28.1 \%$ of total deaths [8]. Until 2003, there were only 418 beds in the palliative care units (PCUs) within the nation. According to Dr. Y. L. Lai, only $16.3 \%$ of patients who died from cancer received care from PCUs [14]. This phenomenon indicates that PCUs are not sufficiently available in Taiwan. In other words, the majority of terminal cancer patients are treated in nonpalliative care units. Our research has shown that $96.9 \%$ of nurses in the gastroenterology (GI), general surgery (GS), and intensive care unit (ICU) have had experience caring for terminal cancer patients, emphasizing the universality of non-palliative care for terminal cancer patients in Taiwan [13]. In modern medicine, the level and quality of care that terminally ill patients receive in PCUs are dramatically different from care received in other units. The PCU focuses on the physical and spiritual comfort of patients, as well as providing attentive care to families. Nonpalliative care units, however, place more emphasis on treatment of disease at different stages and other existing health concerns. Therefore, misplacement of terminal cancer patients in non-palliative care units can actually expose patients to non-beneficial or even harmful treatment measures, with artificial nutrition and hydration (ANH) as a prime example. Normally, it is recognized that ANH can provide advantages such as prolonging life and enhancing patients' physical strength. Some studies suggested that dehydration may contribute to an agitated delirium or terminal restlessness that could be eased by gentle hydration $[3,10,11]$.

Sensation of thirst is a frequent symptom in terminal cancer patients and is associated with dehydration. Morita's study showed that no significant correlations were observed between the visual analogue scale score for thirst and the laboratory tests, which defined biochemical dehydration, such as total protein, blood urea nitrogen, creatinine, sodium, osmolality, hematocrit, and atrial natriuretic peptide [21]. Ellershaw's study showed no statistical significance between the level of hydration and the symptoms form dehydration [9]. Moreover, no evidence that suggested that aggressive nutrition therapy can improve the quality of life. Chiu's study showed that ANH has no significant influence on survival [7]. However, an increasing number of literature reviews have suggested that $\mathrm{ANH}$ is a futile treatment and even brings more harm than good to terminal cancer patients, such as inducing anasarca or pulmonary edema $[4,6,18,20,23,25]$. Zerwekh, a PCU nurse, first realized this in 1983 and published a paper on the disadvantages of ANH and the possible advantages of dehydration in terminally ill patients based on her clinical experience [33]. In 1988, Printz proved that $\beta$-endorphin secretions can be enhanced by starvation in an experiment with a rat model [26]. In 1989, Andrews and Levine reported a study focusing on PCU nurses that showed $71 \%$ of the nurses agreed that reduction of fluid intake can reduce vomiting, $51 \%$ agreed that discontinuation of providing fluid can alleviate the sense of choking and suffocation, and $53 \%$ agreed that terminal dehydration was beneficial to terminally ill patients [1]. It was not until recently that case studies showed that patients passed away peacefully without ANH or from the withdrawal of ANH [27]. Despite these facts, this concept is still relatively unacceptable to the medical professional. Research has shown that medical professionals are biased toward providing ANH $[2,17,19,28]$. Chiu et al. have indicated that the medical professional plays an important role in influencing patients and families about the use of ANH [6]. Nevertheless, our study has shown that nurses lack general knowledge of $\mathrm{ANH}$, resulting in the tendency to provide ANH to terminal cancer patients [13]. This phenomenon led us to conduct research about the educational intervention to non-palliative care unit nurses regarding supplying ANH to terminal cancer patients. Results of this research may reinforce the need to enhance nurses' knowledge and modify their attitudes and behavioral intentions, subsequently providing appropriate knowledge and aiding medical decisions made by patients and their families.

\section{Materials and methods}

\section{Subjects}

A quasi-experimental design was adopted. A structured questionnaire evaluated the effects of educational intervention. From April to June 2005, 88 nurses were enrolled in the GI, GS, and ICU of Taipei Veterans General Hospital in Taiwan. These nurses were randomly assigned into experimental and control groups in equal numbers. Before the study was conducted, the research plan was presented to an Institutional Review Board of Taipei Veterans General Hospital where the study was to take place. After evaluation and approval, the study was formally initiated.

\section{Measurements}

The structured questionnaire includes the following four parts: demographic characteristics, knowledge of providing ANH to terminal cancer patients (15 items), attitudes (17 items), and behavioral intentions (two items). Demographic characteristics included gender, age, education, and experience in caring for terminal cancer patients, experience with $\mathrm{ANH}$ given to terminal cancer patients cared in the past 
year, and perceptions of physical and psychological comfort level of terminal cancer patients in receiving ANH (the scale was $1-10$ points from very uncomfortable to very comfortable). The first draft of the questionnaire was evaluated by expert opinion of four doctors and two nurses, with a content validity index of 0.93 . The validity and reliability of other parts were presented as follows:

1. Knowledge of providing ANH to terminal cancer patients. The content includes goals of ANH treatment, metabolic mechanism and nutritional supply in terminal cancer patients, and relationships between dehydration, hunger, and comfort in terminally ill patients. The scoring system of this scale was "true (1)" and "false/ unknown (0)". Higher scores would, therefore, reflect greater knowledge of provision of ANH to terminal cancer patients among the nurses assessed. The content and consistency of items were generally acceptable, with a Kuder-Richardson formula 20 value of 0.67 .

2. Attitudes toward behavior $(A B)$. This part examined the perception of nurses regarding the advantages and disadvantages of providing $\mathrm{ANH}$ for terminal cancer patients. This is divided into two categories, belief (Bi) and evaluation (Ei). The measure was 17 items using 5point Likert scales from "strongly disagree/very unimportant (1)" to "strongly agree/very important (5)." Regarding advantages of ANH, reverse scoring was employed. Higher total mean score of attitude $(\mathrm{AB}=$ $\Sigma \mathrm{Bi} \times \mathrm{Ei}$ ) indicates the more appropriate attitude towards not supplying ANH to terminal cancer patients. The scores in attitude $(\mathrm{AB})$ ranged from 1 to 25 points. The Bartlett's test of sphericity (BT) and Kaiser-Meyer-Olkin (KMO) test were administered resulting in a BT value of 604.94 (statistical significance $=0.000$ ) and a KMO value of 0.68 , indicating that exploratory factor analysis may be warranted. The principal component analysis, followed by orthogonal varimax rotation, was then employed to carry out construct validity, with a loading value of 0.4 as a cut point. The last two extracted factors were named "benefits of providing ANH" (eight items) and "burdens of providing ANH" (nine items). Cronbach's $\alpha$ for these factors were respectively 0.79 and 0.85 , showing that items contained in each factor could be used to test the same characteristic. Cronbach's $\alpha$ for the overall questionnaire was 0.72 .

3. Behavioral intentions. This section presented the scenario of a terminal cancer patient facing ANH and subsequently assessing tendencies of the nurses to give or withhold the treatment. The 4-point Likert scales was adopted. Based on the hypothesis of this research, the positive behavioral intentions tended toward withholding ANH to terminal cancer patients. The criteria were established as follows: "very unlikely (4)," "unlikely (3)", "likely (2)", and "very likely (1)." Cronbach's $\alpha$ for two items was 0.67 .

Data collection

After the experimental and control groups completed the pretest, only the experimental group participated in a 50-min lecture. Both groups received a post-test 2 weeks after the experimental group ending of the lecture. We compiled the educational portfolio "Critical thinking of providing artificial nutrition and hydration in terminal cancer patients" through massive and rigorous literature review. The portfolio includes handouts and power-point presentations. The difficulty level of the content was assessed by three clinical nurses and determined to be interpretable. The formatting, color, animation, and configuration of the power-point presentation were produced with guidance from a nurse and an instructor who were all familiar with the computer. A 50-min lecture with slide presentation and handouts was given by the principal investigator. The contents included normal nutrient metabolism (7 min), nutrient metabolism for terminal cancer patients (13 $\mathrm{min})$, and the appropriateness of supplying terminal cancer patients with ANH concerning the benefits and burdens of providing ANH in these patients (30 min).

\section{Statistical analysis}

SPSS 11.0 statistics software package was used for data entry and analysis. A $p$ value of $<0.05$ was defined as statistical significance. Chi-square and Yates' tests examine the homogeneity of the nominal variables and the independent sample $t$ test analyzes homogeneity of continuous variables in the demographic characteristics of experimental and control groups. Normal distribution of the sample was examined with Kolmogorov-Smirnov test. The sample, which was non-normal distribution, adopted nonparametric tests including Mann-Whitney $(\mathrm{M}-\mathrm{W})$ test and Wilcoxon signed ranks test. Before the educational intervention, the nurses' knowledge, attitudes, and behavioral intentions were tested for homogeneity. However, prior to educational intervention, no statistically significant differences were shown between the two groups in demographic characteristics, knowledge, attitudes, and behavioral intentions (Tables 1 and 2).

\section{Results}

Overall profile before educational intervention

Both control and experimental groups were highly homogenous, and, therefore, we discuss their information together. Nurses were all females with a mean age of 30.3 , and $67 \%$ 
Table 1 Homogeneity of demographic characteristics

\begin{tabular}{|c|c|c|c|c|c|c|}
\hline Variable & $\begin{array}{l}\text { Control } \\
(n=44)\end{array}$ & $\begin{array}{l}\text { Experimental } \\
(n=44)\end{array}$ & Total $(n=88)$ & $\begin{array}{l}\chi 2 \text { or } \\
t \text { value }\end{array}$ & $95 \% \mathrm{CI}$ & $\begin{array}{l}p \\
\text { value }\end{array}$ \\
\hline $\mathrm{Age}^{\mathrm{a}}$ & & & & -0.564 & $3.19 \sim 1.78$ & 0.574 \\
\hline Mean (SD) & $30.0(5.8)$ & $30.7(5.9)$ & $30.3(5.8)$ & & & \\
\hline Departments, $n(\%)$ & & & & 0.000 & - & 1.000 \\
\hline ICU & $20(22.7)$ & $20(22.7)$ & $40(45.4)$ & & & \\
\hline GI & $5(5.7)$ & $5(5.7)$ & $10(11.4)$ & & & \\
\hline GS & $19(21.6)$ & $19(21.6)$ & $38(43.2)$ & & & \\
\hline Marital status, $n(\%)$ & & & & 2.520 & - & 0.112 \\
\hline Unmarried & $33(37.5)$ & $26(29.5)$ & $59(67.0)$ & & & \\
\hline Married & $11(12.5)$ & $18(20.5)$ & $29(33.0)$ & & & \\
\hline Experience in caring for terminal cancer patients ${ }^{\mathrm{b}}, n(\%)$ & & & & 0.002 & - & 0.967 \\
\hline Yes & $43(50.0)$ & $40(46.5)$ & $83(96.5)$ & & & \\
\hline No & $1(1.2)$ & $2(2.3)$ & $3(3.5)$ & & & \\
\hline Received palliative-care-related in-service education, $n(\%)$ & & & & 0.119 & - & 0.730 \\
\hline Yes & $19(21.8)$ & $17(19.5)$ & $36(41.4)$ & & & \\
\hline No & $25(28.7)$ & $26(29.9)$ & $51(58.6)$ & & & \\
\hline \multicolumn{7}{|c|}{ ANH used by terminal cancer patients cared for in the past year (multiple choices allowed) } \\
\hline Total parenteral nutrition, $n(\%)$ & & & & 0.000 & - & 1.000 \\
\hline Yes & $32(36.4)$ & $32(36.4)$ & $64(72.7)$ & & & \\
\hline No & $12(13.6)$ & $12(13.6)$ & $24(27.3)$ & & & \\
\hline Peripheral parenteral nutrition, $n(\%)$ & & & & 0.656 & - & 0.418 \\
\hline Yes & $37(42.0)$ & $34(38.6)$ & $71(80.7)$ & & & \\
\hline No & $7(8.0)$ & $10(11.4)$ & $17(19.3)$ & & & \\
\hline Intravenous hydration, $n(\%)$ & & & & 0.723 & - & 0.395 \\
\hline Yes & $38(43.2)$ & $35(39.8)$ & $73(83.0)$ & & & \\
\hline No & $6(6.8)$ & $9(10.2)$ & $15(17.0)$ & & & \\
\hline Nasogastric or nasojejunal feeding, $n(\%)$ & & & & 2.009 & - & 0.156 \\
\hline Yes & $39(44.3)$ & $34(38.6)$ & $73(83.0)$ & & & \\
\hline No & $5(5.7)$ & $10(11.4)$ & $15(17.0)$ & & & \\
\hline Stoma feeding, $n(\%)$ & & & & 0.046 & - & 0.830 \\
\hline Yes & $25(28.4)$ & $24(27.3)$ & $49(55.7)$ & & & \\
\hline No & $19(21.6)$ & $20(22.7)$ & $39(44.3)$ & & & \\
\hline
\end{tabular}

${ }^{a}$ Used independent sample $t$ test

${ }^{\mathrm{b}}$ Used Yates's correction

of them were unmarried. Among all nurses, 83 out of 88 (96.5\%) had previous experience with terminal cancer patients care, but $58.6 \%$ had never received palliativecare-related in-service education. In nurses' experiences of giving ANH to terminal cancer patients in the past year, $80.7 \%$ were peripheral parenteral nutrition, $83.0 \%$ were intravenous hydration, $83.0 \%$ were nasogastric or nasojejunal feeding, and $72.7 \%$ were total parenteral nutrition (Table 1). In addition, $78.5 \%$ of nurses had been requested by the terminal cancer patients to give nutritional support, and $93.1 \%$ of nurses had been requested by the family of terminal cancer patients to give nutritional support. The physical comfort level of terminal cancer patients in receiving $\mathrm{ANH}$ was perceived to be $4.95(\mathrm{SD}=1.45$, range 1-10) by the nurses; the psychological comfort level was perceived to be $6.20(\mathrm{SD}=1.65$, range $1-10)$.

Regarding knowledge of provision of ANH for terminal cancer patients, the mean score among nurses was 6.24

Table 2 Homogeneity of knowledge, attitudes, and behavioral intentions of 2 groups

\begin{tabular}{lcccc}
\hline Variable & Control $(n=44)$ & Experimental $(n=44)$ & $t$ value or $z$ value & $95 \%$ CI \\
\hline Knowledge $^{\mathrm{a}}$, mean (SD) & $5.68(2.62)$ & $6.80(3.11)$ & $z=-1.694$ & - \\
Attitudes, mean (SD) & $10.49(1.61)$ & $10.65(2.03)$ & $t=-0.404$ & $-0.94 \sim 0.62$ \\
Behavioral intentions $^{\mathrm{a}}$, mean (SD) & $1.58(0.44)$ & $1.67(0.42)$ & $z=-0.886$ & 0.687 \\
\hline
\end{tabular}

${ }^{\mathrm{a}}$ Used M-W test 
Table 3 Comparison of mean scores of pretest and post-test within single group

\begin{tabular}{|c|c|c|c|c|c|}
\hline Variable & Pretest & Post-test & $t$ value or $z$ value & $95 \% \mathrm{CI}$ & $p$ value \\
\hline \multicolumn{6}{|l|}{ Knowledge $^{\mathrm{a}}$} \\
\hline Control, mean (SD) & $5.68(2.62)$ & $5.21(2.58)$ & $z=-1.517$ & - & 0.129 \\
\hline Experimental, mean (SD) & $6.80(3.11)$ & $10.96(2.95)$ & $z=-5.255$ & - & 0.000 \\
\hline \multicolumn{6}{|l|}{ Attitude } \\
\hline Control, mean (SD) & $10.49(1.61)$ & $10.66(1.42)$ & $t=-0.774$ & $-0.61 \sim 0.27$ & 0.443 \\
\hline Experimental, mean (SD) & $10.65(2.03)$ & $12.79(2.57)$ & $t=-5.191$ & $-2.96 \sim-1.31$ & 0.000 \\
\hline \multicolumn{6}{|l|}{ Behavioral intentions $^{\mathrm{a}}$} \\
\hline Control, mean (SD) & $1.58(0.44)$ & $1.61(0.47)$ & $z=-0.050$ & - & 0.960 \\
\hline Experimental, mean (SD) & $1.67(0.42)$ & $1.97(0.36)$ & $z=-3.274$ & - & 0.001 \\
\hline
\end{tabular}

${ }^{\mathrm{a}}$ Used Wilcoxon signed ranks test

( $\mathrm{SD}=2.91$, range $0-15$ ), with an accurate answer rate of $41.6 \%$. Only two items had an accurate answer rate above $60 \%$; these items were "artificial nutrition replacement can improve hunger status in all terminally ill patients" (69.3\%), and "artificial hydration can improve the sensation of mouth dryness and thirst in all terminally ill patients" $(61.4 \%)$. On the other hand, the two items with the lowest accurate answer rate were "ketoacidosis as a consequence of aphagia often results in an increase of physical pain in terminally ill patients" (9.1\%) and "in terminal cancer patients, chronic starvation results in lipolysis, with subsequent ketoacidosis and anorexia, therefore, nutritional support such as nasogastric feeding should be provided in order to improve malnutrition resulting from anorexia" $(17.0 \%)$. Attitude toward supplying terminal cancer patients with ANH had a mean score of 10.57 (SD=1.91, range 125 ), which shows the negative attitude of nurses toward being inclined to supply ANH to terminal cancer patients. In benefits of $\mathrm{ANH}$, a mean score of $6.31(\mathrm{SD}=2.13)$ was obtained. The highest mean score was "ANH can prolong the life of terminally ill patients (10.58)," indicating that nurses seemed to disagree that ANH prolonged the life of terminally ill patients. In burdens of ANH, a mean score of $14.46(\mathrm{SD}=3.30)$ was obtained, indicating that nurses were aware of various disadvantages of ANH. Among all disadvantages, three received higher scores: "placement of invasive catheters increases the risk of infection in terminally ill patients (16.14)," "in terminally ill patients, fluid overload is likely to result in pleural effusion, pulmonary edema (15.87)," "fluid overload, such as ascites and peripheral edema, can occur in terminally ill patients receiving intravenous infusions (15.64)." The mean score of the behavioral intention to supply terminal cancer patients with $\mathrm{ANH}$ was $1.63(\mathrm{SD}=0.43$, range $1-4)$, indicating that nurses had a tendency to give ANH to terminal cancer patients. Regarding supplying terminal cancer patients with artificial hydration, $100 \%$ of the nurses said either "likely" (59.1\%) or "very likely" (40.9\%). Regarding supplying terminal cancer patients with artificial nutrition, $98.8 \%$ of the nurses said either "likely" (63.6\%) or "very likely" (35.2\%).

\section{Outcomes of educational intervention}

Knowledge of ANH for terminal cancer patients After educational intervention, the mean score of knowledge had significantly increased. Table 3 shows that the control group had pretest and post-test mean scores of 5.68 and 5.21, respectively, and the experimental group had pretest and post-test mean scores of 6.80 and 10.96, respectively. While the mean score for the experimental group was significantly higher after the educational intervention $(z=-5.255, p<0.001)$, the control group had no significant differences $(p>0.05)$. Furthermore, the mean score change

Table 4 Comparison of mean score changes in 2 groups

\begin{tabular}{|c|c|c|c|c|c|}
\hline Variable & Control $(n=44)$ & Experimental $(n=44)$ & $t$ value and $z$ value & $95 \% \mathrm{CI}$ & $p$ value \\
\hline \multicolumn{6}{|l|}{ Knowledge } \\
\hline Mean score change (SD) & $-0.48(2.72)$ & $4.18(3.24)$ & $t=-7.306$ & $-5.92 \sim-3.39$ & 0.000 \\
\hline \multicolumn{6}{|l|}{ Attitudes } \\
\hline Mean score change (SD) & $0.18(1.44)$ & $1.86(2.25)$ & $t=-4.165$ & $-2.48 \sim-0.87$ & 0.000 \\
\hline \multicolumn{6}{|l|}{ Behavioral intentions ${ }^{\mathrm{a}}$} \\
\hline Mean score change (SD) & $0.03(0.56)$ & $0.30(0.52)$ & $z=-1.943$ & - & 0.052 \\
\hline
\end{tabular}

Mean score change $=($ mean score of post-test $)-($ mean score of pretest $)$

${ }^{\mathrm{a}}$ Used $\mathrm{M}-\mathrm{W}$ test 
of knowledge of the experimental group was higher than the control group ( $t=-7.306, p<0.001$; Table 4). This represents the increase in knowledge of ANH for terminal cancer patients after educational intervention.

Attitude towards supplying terminal cancer patients with $A N H$ After educational intervention, the attitude of nurses regarding provision of ANH to terminal cancer patients significantly increased. Table 3 shows that the control group had pretest and post-test mean scores of 10.49 and 10.66 , respectively, and the experimental group had pretest and post-test mean scores of 10.65 and 12.79 , respectively. The experimental group had a much higher mean score after the educational intervention as compared to the mean score of pretest $(t=-5.191, p<0.001)$; the control group had no significant difference $(p>0.05)$. Additionally, the mean score change of attitude of the experimental group was higher than that of the control group $(t=-4.165, p<0.001$; Table 4), indicating a more positive trend in attitude towards supplying ANH to terminal cancer patients after educational intervention.

Behavioral intentions toward supplying terminal cancer patients with ANH After educational intervention, nurses showed an improvement of mean score for behavioral intentions. Table 3 shows that the mean scores of pretest and post-test were 1.58 and 1.61, respectively, for the control group and were 1.67 and 1.97, respectively, for the experimental group. The post-test mean score for the experimental group was higher than pretest $(z=-3.274$, $p \leqq 0.001$ ), and no significant difference was observed for the control group $(p>0.05)$. The mean score changes of behavioral intentions in both groups were not significant $(z=-1.943, p>0.05$; Table 4).

\section{Discussion}

Among all nurse subjects, $96.5 \%$ had experience caring for terminal cancer patients. Among patients being cared for in the past year, over $80 \%$ had received peripheral parenteral nutrition, intravenous hydration, and nasogastric or nasojejunal feeding, and more than $70 \%$ of patients had received total parenteral nutrition. This indicates that nurse subjects had abundant experience prior to this research. However, research results show that nurses seemed to disagree that ANH benefits in prolonging life of the terminal cancer patients. Instead, they are concerned about the known burdens of $\mathrm{ANH}$, especially that infection rates increased with the use of invasive catheters for terminal cancer patients and that anasarca or pulmonary edema, ascites, and peripheral edema are induced by excessive fluid. This demonstrates that nurses seem to realize the disadvantages and inappropriateness of giving ANH to terminal cancer patients, which could be due to their accumulation of direct care and clinical experience with terminal cancer patients. Despite possessing experience in caring for terminal cancer patients, the lack of scientific and evidence-based knowledge towards ANH resulted in a tendency for nurses to continue to supply ANH for these patients. Wurzbach has argued that knowledge deeply influences the clarity of ethics and moral decisions [32]. Thus, sufficient knowledge is a crucial element in making ethical decisions. Domestic research has shown that internship and palliative care classes can dramatically increase the knowledge of palliative care for medical students. However, the concept of not supplying ANH to terminally ill patients was hard for medical students to accept despite their attendance in classes $[5,30]$. The same effects have been observed among nursing students; palliative care knowledge did not influence their ethical agreement toward withholding $\mathrm{ANH}$ for terminal patients [12]. Therefore, aside from clinical experience with palliative care, specialized knowledge towards providing ANH to terminal cancer patients is also necessary. Our educational intervention has produced overall changes in the knowledge, attitudes, and behavioral intentions of nurses regarding supplying ANH to terminal cancer patients. However, mean score changes of the behavioral intention between two groups were not significant. We infer that it can be related to the difference in food culture and family culture within Taiwanese society. "Food comes first for people", "eating is as important as the emperor", and "having food is lucky" is the food culture of Taiwan for a long time. This culture has been subconsciously affecting the Taiwanese. To nurses of Taiwan, they generally assume that "providing ways to eat is the most fundamental care to terminal cancer patients." The idea is similar to the Japanese doctors' belief of "intravenous nutrient being the fundamental care" [19] or the American medical students' belief that "nutrients and hydration are the minimal comfort to patients" [31]. Taiwanese families believe that "requesting nutritional supports for patients is their obligations", and patients believe that "accepting nutritional supports is the responsibility of being a good patient". In addition, a Taiwanese tradition of "not becoming a starving soul after death and affecting one's later generations" often leads to families requiring that ANH be administered by the medical professionals [7]. When this happens, the medical professionals almost always cooperate with the request. In the research, $100 \%$ and $98.8 \%$ of nurses would be providing artificial hydration and artificial nutrition, respectively. This represents the major effect of Taiwanese culture on supplying ANH to terminal cancer patients. Although nurses can accept the fact that ANH has disadvantages that may make patients physically uncom- 
fortable, they claim that ANH has a mentally comforting effect, which appears to concur with scholars who indicate that ANH increases mental support and social significance to the patient $[2,15,16]$. Moreover, affected by familism and paternalism in Chinese culture, families and parents dictate over individuals, therefore, stripping patients of their autonomy [29]. In this research, nurses suggested that when supplying $\mathrm{ANH}$, one should especially consider the autonomy of the patient. However, the patient often loses his or her right to make the decision in a society where the family is the primary decision-making unit. In our research, 93.1\% of nurses have encountered the situation where families of terminal cancer patient make the request to use nutritional support. This is similar with Japan, which also shares the oriental culture where families participate in more than $90 \%$ of the decision making regarding administering ANH to terminal cancer patients [22]. It is different from the study of Israeli where family's involvement in decision-making process to intravenous therapy is only $13 \%$ [24]. This demonstrates the strong influence of families in the medical decision process for terminal cancer patients in oriental culture.

\section{Conclusion}

The experimental group showed significant improvement in mean scores of knowledge, attitudes, and behavioral intentions towards ANH to terminal cancer patients. Furthermore, mean score changes in "knowledge" and "attitude" between experimental and control groups were statistically significant, indicating the efficacy of educational intervention. However, mean scores of changes of behavioral intentions between two groups were not significant; reflecting that the behavioral intentions of nurses are also affected by other variables. These variables may include the ethics and morality of the Taiwanese culture and the powerful role of families. We recommend integrating these additional topics into school and in-service education in order to devise long-term training schemes and domestic guidelines.

Acknowledgments The authors thank the nurses of gastroenterology, general surgery, and intensive care unit of Taipei Veterans General Hospital for their full support.

\section{References}

1. Andrews MR, Levine AM (1989) Dehydration in the terminal patient: perception of hospice nurses. Am J Hosp Care 6:31-34

2. Asch DA, Christakis NA (1996) What do physicians prefer to withdraw some forms of life support over others? Intrinsic attributes of life-sustaining treatments are associated with physicians' preferences. Med Care 34:103-111

3. Bruera E, Franco JJ, Maltoni M, Watanabe S, Suarez-Almazor M (1995) Changing pattern of agitated impaired mental status in patients with advanced cancer: association with cognitive monitoring, hydration, and opioid rotation. J Pain Symptom Manage 10:287-291

4. Burge FI, King DB, Willison D (1990) Intravenous fluids and the hospitalized dying: a medical last rite? Can Fam Physician $36: 883-886$

5. Chang HH, Hu WY, Chiu TY et al (2003) An interventional study assessing palliative care learning amongst junior medical students undertaking the course "the human side of medicine". J Med Educ $7: 150-160$

6. Chiu TY, Hu WY, Chuang RB et al (2004) Terminal cancer patients' wishes and influencing factors toward the provision of artificial nutrition and hydration in Taiwan. J Pain Symptom Manage 27:206-214

7. Chiu TY, Hu WY, Chuang RB, Chen CY (2002) Nutrition and hydration for terminal cancer patients in Taiwan. Support Care Cancer 10:630-636

8. Department of Health, Executive Yuan, Taiwan, R.O.C. 2006 Statistical summary of the main causes of death in Taiwan. Available at http://www.doh.gov.tw/statistic/index.htm. Accessed Sep 23, 2007

9. Ellershaw JE, Sutcliffe JM, Saunders CM (1995) Dehydration and the dying patient. J Pain Symptom Manage 10:192-197

10. Fainsinger R, Miller MJ, Bruera E, Hanson J, Maceachern T (1991) Symptom control during the last week of life on a palliative care unit. J Palliat Care 7:5-11

11. Fainsinger RL, Bruera E (1997) When to treat dehydration in a terminally ill patient? Support Care Cancer 5:205-211

12. Hu WY, Tseng CN, Wang Y, Ueng RS (2004) The effects of clinical practice program toward palliative care for nursing students' education in school of nursing. Taiwan J Hosp Palliat Care 9:1-20

13. Ke LS, Chiu TY, Lo SS, Hu WY (2007) Knowledge, attitudes, and behavioral intentions of nurses toward providing artificial nutrition and hydration for terminal cancer patients in Taiwan. Cancer Nurs 31:67-76

14. Lai YL (2004) Hospice palliative care in Taiwan. Formosan J Med 8:653-656

15. Lynn J, Childress JF (1983) Must patients always be given food and water? Hastings Cent Rep 13:17-21

16. Mc Inerney F (1992) Provision of food and fluids in terminal care: a sociological analysis. Soc Sci Med 34:1271-1276

17. Micetich KC, Steinecker PH, Thomasma DC (1983) Are intravenous fluids morally required for a dying patient? Arch Intern Med 143:975-978

18. Morita T, Hyodo I, Yoshimi T et al (2005) Association between hydration volume and symptoms in terminally ill cancer patients with abdominal malignancies. Ann Oncol 16:640-647

19. Morita T, Shima Y, Adachi I (2002) Attitudes of Japanese physicians toward terminal dehydration: a nationwide survey. $\mathrm{J}$ Clin Oncol 20:4699-4704

20. Morita T, Tei Y, Inoue S, Suga A, Chihara S (2002) Fluid status of terminally ill cancer patients with intestinal obstruction: an exploratory observational study. Support Care Cancer 10:474-479

21. Morita T, Tei Y, Tsunoda J, Inoue S, Chihara S (2001) Determinants of the sensation of thirst in terminally ill cancer patients. Support Care Cancer 9:177-186

22. Morita T, Tsunoda J, Inoue S, Chihara S (1999) Perceptions and decision-making on rehydration of terminally ill cancer patients and family members. Am J Hosp Palliat Care 16:509-516

23. Musgrave CF, Bartal N, Opstad J (1995) The sensation of thirst in dying patients receiving i.v. hydration. J Palliat Care 11:17-21 
24. Musgrave CF, Bartal N, Opstad J (1996) Intravenous hydration for terminal patients: what are the attitudes of Israeli terminal patients, their families, and their health professionals? J Pain Symptom Manage 12:47-51

25. Philip J, Depczynski B (1997) The role of total parenteral nutrition for patients with irreversible bowel obstruction secondary to gynecological malignancy. J Pain Symptom Manage 13:104-111

26. Printz LA (1988) Is withholding hydration a valid comfort measure in the terminally ill? Geriatrics $43: 84-88$

27. Smith SA (1997) Controversies in hydrating the terminally ill patient. J Intraven Nurs 20:193-200

28. Solomon MZ, O’Donnell L, Jennings B et al (1993) Decisions near the end of life: professional views on life-sustaining treatments. Am J Public Health 83:14-23
29. Tsai PJ (2004) The ethical dilemma and social work ethical justification for terminal medical communication. Taiwan J Hosp Palliat Care 9:141-152

30. Tsai SL, Hu WY, Chiu TY et al (2004) The knowledge of palliative care amongst senior medical students undertaking the course "family, society and medicine". Formosan J Med 8:313322

31. Weissman DE, Ambuel B, Norton AJ, Wang-Cheng R, Schiedermayer D (1998) A survey of competencies and concerns in endof-life care for physician trainees. J Pain Symptom Manage 15:82-90

32. Wurzbach ME (1995) Long-term care nurses' moral convictions. J Adv Nurs 21:1059-1064

33. Zerwekh JV (1983) The dehydration question. Nursing 13:47-51 